\title{
Improving feedback from outpatient medical appointments attended by escorted psychiatric patients in the North London Forensic Service
}

\author{
Kathleen McCurdy, Anna Croxford
}

Barnet, Enfield and Haringey Mental Health Trust

\begin{abstract}
It is well established that patients with mental illness are known to have a high level of morbidity and mortality compared to the general population. This is particularly prominent in long-stay psychiatric patients, such as those in secure settings. The Royal College of Psychiatrists recommends that psychiatrists should promote the physical health of their patients and liaise with other specialties. However, there is evidence that communication between psychiatry and other specialties is poor.

A survey was carried out at the North London Forensic Service in June 2014. This looked at the views of clinical staff about the frequency and quality of feedback obtained when inpatients attend outpatient hospital appointments at local general hospitals. This survey highlighted the general perception among staff that feedback is poor, with $68.43 \%$ of respondents saying that they were "very unsatisfied" or "unsatisfied" with the level and quality of feedback. Clinical staff felt that many patients who attended hospital outpatient appointments, even when escorted by staff, returned with little or no feedback. This was confirmed by a baseline audit across 3 wards showing that details of the appointment (date, time, hospital, and specialty) were only documented in $54.5 \%$ of cases and the content of the appointment documented in even fewer cases.

A form was designed by junior doctors that provided a simple framework of 6 questions to be answered at the outpatient clinic about the problem, diagnosis, and further actions needed. This was introduced and its impact assessed with a 3-month and 6-month audit of electronic notes, as well as a follow-up survey after 6 months. The audit showed significant improvement in the quality of feedback about the appointment at both the 3-month and 6-month point. The follow-up survey showed that $70 \%$ of respondents were aware of the form and $100 \%$ of those who were aware of the form had used it at least once and found it helpful. The general satisfaction level improved, but remained low, with $40 \%$ of respondents saying that they were "very unsatisfied" or "unsatisfied" with the level and quality of feedback.
\end{abstract}

This QIP shows that the outpatient appointment form is a useful and effective tool when staff know about it and use it. We plan to look at the barriers to using the form and consider future solutions to the need for increased engagement with nursing staff stakeholders in future PDSA cycles.

\section{Problem}

The North London Forensic Service, based at Chase Farm Hospital, provides inpatient beds in both low secure and medium secure environments for patients from the London boroughs of Camden, Islington, Barnet, Enfield, and Haringey. Many of these patients have chronic, disabling mental illnesses with multiple psychiatric and physical co-morbidities that require input from many different specialties. Frequently our patients need to attend various hospitals across London for appointments with clinicians from many different specialties and are accompanied to these by ward staff.

However, anecdotal evidence suggests that often the verbal feedback brought back for the psychiatric multidisciplinary team (MDT) is inadequate and it can take weeks for a clinic letter to reach the ward. In this way patient care is negatively affected as there is a significant delay in implementing recommendations made by the medical or surgical team, The MDT is not always fully aware of a patient's physical health and the junior doctors and nursing staff spend a significant amount of time speaking to medical secretaries and hospital doctors to gather information and therefore taking time out of clinical work.
The aim of this project is ultimately to improve the communication between medical specialties and the forensic psychiatry team in order to enhance patient care. Specifically we wanted to gain prompt and accurate feedback from outpatient appointments from the accompanying staff.

\section{Background}

It is well established that patients with mental illness are known to have a high level of morbidity and mortality compared to the general population (1). For example, it has been found that levels of cardiovascular disease, respiratory disease, and malignancy are significantly higher in those with mental illness (2). A study of patients known to psychiatric secondary care in southeast London showed that life expectancy is shortened by between 10 and 17 years in those with mental illness. (3)

This is very relevant to patients in secure settings who tend to be inpatients for a longer period of time and have high rates of smoking, obesity, and related health problems (4). A study looking at the physical health of patients in a medium secure hospital in Hampshire noted that the rates of smoking was over three times as 
prevalent in this population than in the general population $(84 \%$ compared to $27 \%$ ), that patients tended to gain weight whilst inpatients, and spoke of a need to train nurses and multidisciplinary team members about the physical health needs of this population (5).

The myriad reasons why mental health patients have poor physical health have been widely studied in the literature. It is estimated that around $60 \%$ of the excess mortality in people with mental illness is avoidable and there are many reasons for this (6); Side effects of medications prescribed for mental illness, patient lifestyle factors, and social issues such as access to healthcare all have a part to play (7). For example, atypical antipsychotic medication can lead to weight gain, diabetes, and raised cholesterol 8 ) and this is compounded by an increased level of smoking, substance misuse, and lack of exercise in the patient group (9). Patients with mental health illness are also more likely to become non-compliant with their physical health medications and are less likely to attend followup clinics for their physical problems. The separation of mental and physical health services, the stigma associated with having a mental health diagnosis and the difficulty some patients have in understanding and implementing the health care advice given are practical reasons why people with mental illness have high morbidity and mortality (10).

There has been much focus recently on improving physical health in people with mental illness and this was one of the key issues explored in the 2014 Chief Medical Officer's (CMO) annual report on public mental health. (11) Over the past few years there have been many new initiatives and measures designed to help improve physical health in those with serious mental illness. Following NICE recommendations (12) the introduction of "smoke-free hospitals" in many mental health trusts, including South London and the Maudsley (SLAM) and Camden and Islington NHS Foundation Trust (13), has encouraged patients to consider stopping smoking. The Commissioning for Quality and Innovation (CQUINs) framework links physical health to payments thus increasing the incentive to actively assess and manage our patients with physical health problems.(14) Practically measures such as the Lester Framework, a framework for assessing and treating cardiometabolic risk in those with psychosis, gives clinical teams clear guidance on how to assess and manage these physical health risks (15).

A Royal College of Psychiatrists scoping group report, entitled Physical Health in Mental Health (16), suggests that "health professionals should enable people with mental disorders and disabilities to have the same standard of physical healthcare as other citizens". They advise that "psychiatrists should promote the physical health of their patients and, where appropriate, refer patients to colleagues in other medical specialties". There is some evidence that communication between psychiatry and other specialties is poor, although this mainly focuses on the primary care interface (17). Although the focus is on doctors to promote the physical health of their patients there is also an expectation that nursing staff are aware of physical health issues. However in a study by Rylance et al (18) a qualitative analysis showed that nursing staff on acute psychiatric wards "took minimal responsibility for physical health monitoring" and felt that their role was one of referring on if there were concerns about a patient's physical health.

\section{Baseline measurement}

In June 2014 a questionnaire was distributed to all staff in working on the forensic psychiatry inpatient wards on the Chase Farm site to try and understand the scale of the problem with poor feedback from outpatient appointments and the factors influencing this. The questionnaire was designed using input from junior doctors, discussion at monthly junior doctors' forum, and at clinical governance meetings involving the MDT and ward staff. During these meetings it was suggested that often escorting staff do not know what questions to ask the doctors or what the important points are that the team would like to know, some staff feel that they should be concentrating on mental health rather than physical health or are simply too busy on their shifts to document details about the content of medical appointments. Questionnaires were formulated and distributed by the authors in electronic form to all clinical staff working at the service to assess the scale and perception of the problem across different staff groups. Please see supplementary file for the structure and results of the initial survey.

19 responses were gained from the questionnaire, evenly spread between doctors and nursing staff. The respondents said that they send patients for outpatient appointments up to 2-3 times per week. However, nearly half of respondents $(47.63 \%)$ estimated that less than a third of their patients returned from appointments with any feedback about the content of the medical appointment and only 1 respondent felt that all appointments were fed back on adequately. $42.11 \%$ of respondents said that on average a written letter from medical appointments took over a month to arrive on the ward. $68.43 \%$ said that they were "very unsatisfied" or "unsatisfied" with the level and quality of feedback. $89.47 \%$ of respondents felt that introducing a short form with a few simple, general questions that escorting staff should clarify with the outpatient team who the patient is seeing would be helpful. Those who did not feel this was helpful gave various reasons including "feedback is already good", "it would take too much time" and "I don't feel it is part of the role of escorting staff to feedback on medical issues".

A retrospective audit of the electronic patient notes (RiO notes) of all patients across 3 inpatient wards was used to gain objective baseline measurements of the scale of the problem. We looked at a 3 month period from April to June 2014 and identified all instances when a patient attended an outpatient hospital appointment. All entries by doctors were manually searched for evidence of clinic appointments or attendance and keywords were used to search nursing notes. The entry made by the staff escort was identified and assessed for whether it contained a) details of the appointment (that is which hospital, which department, which healthcare specialist), b) a description of the content of the appointment (what investigations were done, what the presenting complaint was, whether a diagnosis was made), and c) the outcome of the appointment (whether the patient was discharged or due to be followed up, any changes to treatment plan).

The search identified 11 hospital outpatient appointments that were attended in that time. In 6 cases (54.5\%) details of the appointment 
were documented. In 3 cases (27.2\%) a description of the content of the appointment was documented and in 5 cases (45.4\%) the outcome was documented.

See supplementary file: ds4875.pptx - "Results of Initial Survey on Feedback from Medical Appointments"

\section{Design}

A number of ideas were considered for how to improve on the amount and quality of feedback including putting up posters on the wards, emailing staff, reminding staff in clinical governance and ward rounds, and having teaching sessions. However, it was felt that many of these interventions may not be sustainable in the longer term once the junior doctors involved in the project had rotated through. Therefore we felt the introduction of a short form with 6 simple questions to ask at each medical appointment would be appropriate and this was supported by $89.47 \%$ of responded in the baseline survey.

The information requested by the form included basic information such as patient name, date of appointment, the service, and the clinician it was with. The 6 points of information requested were as follows. Please note that the examples in brackets were added later, in response to suggestions of the focus group in PDSA cycle 1 :

1. What was the purpose of appointment / patient symptoms (eg abdominal pain, poor eyesight)

2. What is the problem or diagnosis? (eg irritable bowel, diabetic complications, unknown)

3. Are further investigations needed? Which ones? When and by whom? (eg colonoscopy, x-ray, blood tests)

4. What is your advice regarding the management of the problem? (eg lifestyle changes, blood sugar monitoring changes in medication, surgery, physiotherapy)

5. Will there be any follow up? When will this be and with whom?

6. Do the psychiatric team need to do anything, and if so what?

\section{Strategy}

In PDSA cycle 1, the initial trial period, forms were distributed to teams across 3 wards. Emails were sent to the appropriate ward managers with the form asking for copies to be distributed, copies were placed on wards and it was highlighted in ward rounds. Forms were placed on the wards with an attached sheet with a brief explanation of how the form would be useful to staff and patients, how it should be used. Junior doctors placed copies of the forms in the ward diaries where patient appointments were written and reminded staff to use the forms.

An audit showed that over the course of 2 weeks the forms were used on 3 occasions. In no case was the form uploaded onto RiO, but in all 3 cases the questions on the form were used to guide information documented on $\mathrm{RiO}$. In all 3 cases details of the appointment, content of the appointment, and outcome were commented on in the notes. A focus group with the 4 junior doctors in the relevant teams was held to discuss the success of the form. This elicited that the junior doctors rated themselves as very satisfied with the data captured when the forms were used and felt that they were able to feed the information back to the team at the next ward round and act promptly to make any changes to treatment necessary. A few amendments were suggested including the rewording of ambiguous questions and the inclusion of examples to clarify the meaning of the question. It was highlighted that the doctors had to be active in reminding staff to use the form for example reminding staff and handing staff the form.

In PDSA cycle 2 the form was sent via email to the doctors and ward managers across the service and they were asked to use the forms. The topic was again discussed at junior doctors' meeting at the beginning of the next rotation of doctors to highlight the use of the form to trainees new to the service and asking them to encourage their use. An audit of the electronic notes of 3 wards was again performed at the 3 month point and the 6 month point after the form was introduced. After 6 months a follow-up survey was sent to all clinical staff across the service asking their opinions on the form and how effective it had been in addition to the same measurements of satisfaction used in the previous survey.

See supplementary file: ds4872.doc - "Physical Health Appointments Template"

\section{Post-measurement}

Retrospective audit was used at the 3-month follow-up and at the 6-month follow-up point (looking at the 3-month interval between audit points) to objectively assess whether the forms were in use and whether documentation of feedback from medical appointments had improved. This was supplemented by a 6 -month follow-up survey of clinical staff asking their views on the form and their assessment of the quality of feedback.

3-month follow-up audit

11 appointments were attended across the 3 wards. In 0 cases was the form uploaded onto RiO. However, in 8 cases $(72.7 \%)$ the structure of the form was used in the RiO entry. The quality of feedback had markedly improved with $100 \%$ of entries containing details of the appointment, and 9 out of $11(81.8 \%)$ having details of both the content of the appointment and the outcome of the appointment.

\section{6-month follow-up audit}

12 appointments were attended across the 3 wards. In 0 cases was the form uploaded onto RiO. However, in 6 cases (50\%) the structure of the form was used in the RiO entry. The quality of feedback had shown sustained improvement with 10 (83.3\%) of entries containing details of the appointment. 9 entries $(75 \%)$ had details of the content of the appointment and 9 entries $(75 \%)$ had details about the outcome of the appointment. See supplementary file for table and graph of audit data. 
6-month follow-up survey

10 responses were received to the 6 month follow-up survey; $40 \%$ from medical staff and $60 \%$ from nursing staff. All but one of these responses were from people who worked on one or more of the three wards where the form was initially trialled. $70 \%$ of respondents were aware of the form. $100 \%$ of those who were aware of the form had used it at least once and found it helpful. We looked at the reasons why staff did not use the form. Of those who did not use the form $66 \%$ did not know where to find the form and $33 \%$ said that they did not have the time to fill out the form. Nobody responded with finding the form too difficult, feeling that this was not their job or the "other" category.

With regards to satisfaction measurements there was improvement in the amount of feedback received by the team with only $10 \%$ of respondents feeling that less than a third of patients came back with feedback, a marked improvement from baseline. However, $50 \%$ of respondents still felt that "less than $2 / 3$ of the patients returned with feedback". Overall there was an increase of satisfaction with the quality of feedback with only $10 \%$ of respondents feeling "very unsatisfied" and $40 \%$ of respondents feeling either "very unsatisfied" or "unsatisfied". 30\% felt either satisfied or very satisfied. Please see supplementary file for figures.

We used a comment box to try to elicit barriers to using the form that were not captured in the multiple choice portion and to elicit ways in which this intervention could be improved on. The comments centered on improving engagement with nursing staff and ward managers, providing more copies of the form on wards or make it easier to find, and to think about the ways in which the culture could be changed to put more emphasis on physical health, not just mental health. Please see supplementary file for all individual comments.

See supplementary file: ds5075.pptx - "Post-Intervention measurements"

\section{Lessons and limitations}

By implementing this project we have learned a number of lessons that will be important in moving the project forward and continuing to improve feedback from hospital appointments.

One of the main limitations of this project is the small number of responses to our questionnaire despite multiple emails being sent across the wards and it being highlighted at ward meetings. It is notable that in the follow-up survey $90 \%$ of the replies were from people working on the pilot wards. It appears that these teams, where the junior medical staff were actively advocating and enabling the use of the form, were the most enthusiastic in providing feedback. It is important to consider whether people who were enthusiastic in giving feedback are likely to be those interested in the topic or using the forms, and may therefore not be representative of nursing staff as a whole.

Another limitation is that the 3-month and 6-month audits were carried out on the 3 pilot wards, rather than on all the inpatients wards. This means that we cannot extrapolate that the project was successful across the service and can only comment on the uptake and usage on these 3 wards.

This project has highlighted the difficulties in rolling out a new initiative to more than one ward and across different teams and raises an important consideration about ownership. In teams where the junior medical staff, those actively involved in the QIP, were advocating the use of the form the project proved reasonably successful. However we do not know whether it worked on other wards or if it was used at all. The North London Forensic Service houses a large number of patients in many wards and has hundreds of staff across many different teams. Due to this it proved logistically challenging to publicise the project on every ward and with every team and it was impossible to remind all the staff to use a form for each hospital appointment unless a junior doctor was present at the time.

Consideration should be given in the next PDSA cycle as to how to give each team ownership of the project. This could be facilitated by asking consultants to incorporate it into ward round feedback, asking junior doctors to provide teaching on the form and how to use it on each ward, and getting the support of ward managers and ward clerks to replenish the stocks of forms and highlight the expectation to use it. As most wards do not have patients going for medical appointments every week it is going to take a significant amount of time and sustained proactive effort to get the use of the form embedded in the culture of each ward team. A newly appointed senior nurse has been allocated the role of physical health lead for the service. He will be liaised with as one of the key stakeholders in taking the project forward.

Given that the junior doctors change rotation every 6 months and often change trust, it makes it difficult for a project of this size to fully come to completion during the time frame when a doctor is there. Therefore it is important to have a permanent member of staff or a subsequent trainee associated with the project and continuing to implement PDSA cycles until it is sustainable within the culture of the service.

In future the use of paper surveys, in addition to online surveys and feedback from clinical governance, could be used to gather information on clinical team perception of feedback and their views on the form. For future audits about usage of the form it would be useful to have a specific folder on each ward where paper copies of the forms could be filed after use. The use of the folder could be and incorporated into ward round but this would also enable us to do spot audits on the use of the form and the quality of the responses.

In taking this project forward it is important to understand more clearly any resistance to using the form and any barriers in place. Given that many staff still rate their satisfaction with feedback about medical appointments as poor, it would be useful to know why this is and what could be improved on. It may be helpful to have focus groups of nursing staff to explore this more completely; reasons given in the survey why the form might not be used included "it is not my job to feed back about physical health", "it takes too much" 
"sometimes the feedback we get is that the doctor will get in touch with the psychiatry consultant". These responses suggest that maybe staff encounter resistance whilst at the outpatient appointment from hospital practitioners, have anxieties around dealing with physical health matters or are simply overwhelmed by paperwork already. It is important to unpick these elements in more detail to understand the experiences of those who are filling out the forms. The involvement of ward nursing staff, as the major stakeholders in this project, is key to implementing change and improved patient care.

\section{Conclusion}

Many studies have highlighted the poor physical health of psychiatric patient, including those in secure settings, and the Royal College of Psychiatrists advises that "psychiatrists should promote the physical health of their patients" (6). Part of this is liaising with colleagues in other medical specialties to ensure timely diagnosis and treatment of any co-morbidities. It is therefore vital that good communication channels exist between specialties and that there is a change of culture in psychiatric hospitals where patients' physical health is considered as important as their mental health.

Baseline measurements demonstrated that there is a high level of dissatisfaction amongst psychiatrists and ward staff about what feedback we are getting on the physical health of our patients. Baseline measurements showed that across 3 wards there was low quality of feedback from escorting staff. However satisfaction levels appear to have improved after 6 months of the intervention and there was noticeable improvement in the quality of feedback received from medical appointments. Although there may have been variable uptake on the wards, our project shows that some staff are aware of the tool and have used it. When the forms have been used staff have felt that they were useful. This project suggests that when used regularly and appropriately this initiative has the potential to improve the quality and efficiency of feedback to the multi-disciplinary team. However, further PDSA cycles should continue to refine the tool and its implementation as well as consider other alternative methods to engage nursing staff and promote a change in culture.

\section{References}

1. Phelan M, Stradins L, Morrison S. Physical health of people with severe mental illness. BMJ. 2001; 322:443-444.

2. Carney CP, Jones L, Woolson RF. Medical comorbidity in women and men with schizophrenia: a population-based controlled study. J Gen Intern Med 2006;21(11):1133-7.

3. Chang C.K. et al., All-cause mortality among people with serious mental illness (SMI), substance use disorders, and depressive disorders in southeast London: a cohort study. BMC Psychiatry. 10: $p 77$

4. Cormac, I., Ferriter, M., Benning, R., \& Saul, C. Physical health and health risk factors in a population of long-stay psychiatric patients. Psychiatric Bulletin, 29(1), 2005: 18-20.

5. Meiklejohn, C., Sanders, K., \& Butler, S. Physical health care in medium secure services. Nursing standard, 2003;
17(17), 33-37.

6. Hoang U., Goldacre MJ., Stewart R., Avoidable mortality in people with schizophrenia or bipolar disorder in England. Acta Psychiatrica Scandinavice 2013. 127; 195-201

7. De Hert M, Correll CU, Bobes J, Cetkovich-Bakmas M, Cohen D, Asai I, Detraux J, Gautam S, Möller HJ, Ndetei DM, Newcomer JW, Uwakwe R, Leucht S. Physical illness in patients with severe mental disorders. I. Prevalence, impact of medications and disparities in health care. World Psychiatry 2011;10(1):52-77.

8. Robson D, Gray R. Serious mental illness and physical health problems: a discussion paper. Int J Nurs Stud 2007;44:457-66.

9. Lawrence, D., \& Kisely, S. Review: Inequalities in healthcare provision for people with severe mental illness. Journal of psychopharmacology, Lawrence, D., \& Kisely, S. (2010). Review: Inequalities in healthcare provision for people with severe mental illness. Journal of psychopharmacology, 2010; 24(4 suppl), 61-68.

10. O'Brien G, Bullock R, Black S, Cormac I, Jones G, Kerr M, Murphy M, Naylor K, Osborn D, Phelan M, Ramsay R. Physical health in mental health; Final report of a scoping grou". London: Royal College of Psychiatrists 2009. Royal College of Psychiatrists Occasional Paper OP67.

11. Department of Health. Chief Medical Officer (CMO) annual report: public mental health. Available from https://www.gov.uk/government/uploads/system/uploads/att achment data/file/413196/CMO web doc.pdf

12. National Institute for Health and Care Excellence. Smoking Cessation in secondary care: acute, maternity and mental health services, 2013. NICE public health guidance.

13. http://www.slam.nhs.uk/our-services/smokefree http://www.candi.nhs.uk/betternicotinemanagement/c-i-to-gosmokefree-in-march-2015/

14. RCPsych. CQUIN for Mental Health. http://www.rcpsych.ac.uk/workinpsychiatry/qualityimprovem ent/cquin.aspx

15. RCPsych. Lester Adaptation. Available from http://www.rcpsych.ac.uk/PDF/LesterUKAdaptation2014upd ateA5booklet landscape version.pdf

16. O’Brien G, Bullock R, Black S, Cormac I, Jones G, Kerr M, Murphy M, Naylor K, Osborn D, Phelan M, Ramsay R. Physical health in mental health; Final report of a scoping group". London: Royal College of Psychiatrists 2009. Royal College of Psychiatrists Occasional Paper OP67.

17. Durbin J, Barnsley J, Finlayson B, Jaakkimainen L, Lin E, Berta W, McMurray J. Quality of Communication Between Primary Health Care and Mental Health Care: An Examination of Referral and Discharge Letters. The Journal of Behavioral Health Services \& Research 2012; 39: 4: 445-461

18. Rylance, R., Chapman, H., \& Harrison, J. Who assesses the physical health of inpatients? Nurses' perceptions of their role in this area show they are unprepared and unsure of their responsibilities, say Rebecca Rylance and colleagues. Mental Health Practice,2011; 16(2), 14-20.

\section{Declaration of interests}


Nothing to declare.

\section{Acknowledgements}

Dr Laura Samso for her help in designing, implementing and auditing this QIP

Dr Georgios Kafas and Dr Balroop Mann for their involvement in designing and implementing this QIP

Dr Susie Lingwood, Consultant Psychiatrist, Leadership and Service Improvement Tutor for her useful advice

Doctors and nursing staff at North London Forensic Service

\section{Ethical approval}

Ethical approval was not required for this project as per the Medical Research Council Health Research Authority research decision tool guidance. 